\title{
Novel biocompatible transversal pneumatic artificial muscles made of PDMS/PET satin composite
}

\author{
Tomasz Szmechtyk $^{1 *}$, Natalia Sienkiewicz ${ }^{1}$, Katarzyna Koter ${ }^{2}$, Agnieszka Kobierska ${ }^{2}$, \\ Krzysztof Strzelec ${ }^{1}$ \\ ${ }^{1}$ Lodz University of Technology, Institute of Polymer and Dye Technology, Lodz, Poland \\ ${ }^{2}$ Lodz University of Technology, Institute of Machine Tools and Production Engineering, Lodz, Poland \\ "Corresponding author: e-mail: tomaszszmechtyk@gmail.com
}

\begin{abstract}
In this study novel transversal pneumatic artificial muscles (TPAM), made from composite - poly(dimethylsiloxane) (PDMS) matrix membrane and poly(ethylene terephthalate) (PET) satin reinforcement, are presented. Miniature TPAM consists of a flexible internal braid (IB) reinforcing the membrane and the external braid (EB). EB, with fibers arranged transversely to the IB, is placed laterally. Differently prepared TPAMs were tested for their effectiveness as actuators for robot drive and the PDMS/PET composite suitability was evaluated for applications in human gastrointestinal tract (chemical resistance, thermal characteristic). FT-IR spectra of the composite were compared for study PDMS impregnation process of PET satin and effect of immersion in selected solution. The composite shows outstanding biocompatibility and the muscles have competitive static load characteristics in comparison with other pneumatic artificial muscles (PAM). These results lead to believe, that in the near future painless examination of the gastrointestinal tract using a secure robot will be possible.
\end{abstract}

Keywords: transversal pneumatic artificial muscles, chemical resistance, thermal characteristic, FT-IR spectroscopy, static load characteristic.

\section{INTRODUCTION}

Drive system is a necessary element of each robot. There are robots with pneumatic, hydraulic or the most widely used electric drive. Pneumatic and hydraulic actuators are used as a second source of energy, however, they also play an important role in the operation of robots. Pneumatic systems consist of three parts: system of preparing air, control system and actuators. Actuators are the pneumatic cylinders, where the pressure energy is produced by the working medium and converted into mechanical energy of linear, angular or curved motion. Interesting type of pneumatic actuator is Pneumatic Artificial Muscle (PAM).

PAM consists of a flexible braid element, which is reinforced by membrane. Transfer of the mechanical power along the muscle is possible because of fittings attached to the membrane. PAMs are actuators operated by inflating working medium, usually compressed air, into the membrane. Expansion of the muscle is caused by inflation of the gas, contraction occurs in result of releasing gas (Fig. 1). It is possible to control the force generated by the muscle by controlling a value of the pressure supplied to the muscle ${ }^{1}$.

The best known and most widely used type of muscle is the McKibben muscle, which is made of inflatable

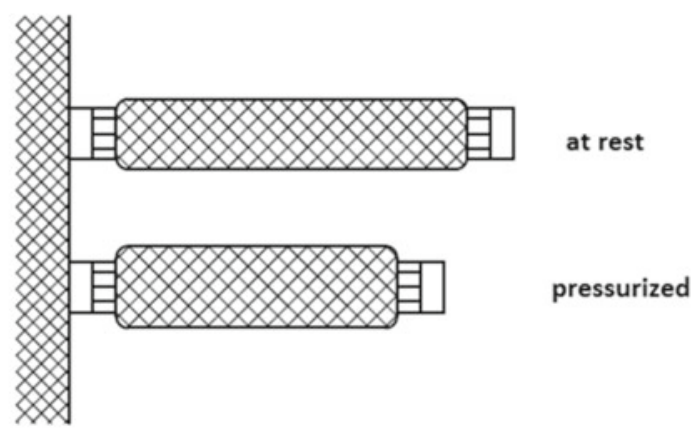

Figure 1. Working of PAM rubber bladder stiffened with braided mesh nylon yarn. Filling muscle with compressed air causes deformation of the membrane, both the radial and longitudinal. The fittings at the ends of each muscle allow to obtain a movement and a pulling force, which depend of muscle contraction. McKibben muscles are able to work at pressures of 0.1-0.5 MPa. The maximum pressure value is determined by the strength of membrane material too high pressure of the working medium can rupture the membrane. The pressure of the working medium will also affect on the force generated while operating muscle - the higher working pressure, the higher values of force are achieved ${ }^{2}$.

Another type of muscles are Pleated Artificial Muscles. These muscles belong to braided muscle group and consist of a membrane with embedded core, which carries a tension. The membrane has plurality of folds arranged in the longitudinal direction. As a result of bringing the working medium to the muscle, the pleated material straightens out and dramatically shortens the muscle, while a large change in diameter is provided. Characteristics of these muscles depend on: the maximum length to minimum diameter ratio, deformation of the membrane material and a set pressure of the compressed air $^{3,4}$.

Micro muscles created by Young Kwun Lee and Isao Shimoyama are based on the design of McKibben muscles. They have been designed in cooperation with Samsung, during the design of the artificial hand. To imitate the movement of a real hand, there were designed micro muscles for the drive, measuring 18 and $30 \mathrm{~cm}$ in length and $1 \mathrm{~mm}$ in diameter. Most attention was paid to the material aspects of braid and membrane. In particular, the structure distinguishes itself by softer material of a core, which allowed the muscle to perform more fluid movements. Additionally, the use of special materials enabled reduction of the muscle, achieving a maximum force of $3.8 \mathrm{~N}$ for contraction at $21 \%$ for a pressure of 6 
bar ${ }^{5}$. The design of the micro muscles is simple and not timeconsuming, what allows the reduction of production costs. Also, it can be a decisive factor while choosing the kind of drive for pneumatic systems ${ }^{6}$. However, the miniaturization of the muscle reduces the shortening degree and the force exerted by the muscle.

Transversal PAMs represent an alternative to the previously used structures. These muscles, patented by the Institute of Machine Tools and Production Engineering at the Faculty of Mechanical Engineering at Lodz University of Technology, can eliminate the imperfections of other versions.

Miniature Transversal PAM (TPAM) consists of a flexible internal braid (IB) reinforcing the membrane and the external braid (EB). EB, with fibers arranged transversely to the IB is placed laterally. While resting, the IB is U-shaped, as shown in Figure 2a. When the gas is inflated to a muscle, the IB bulges and changes a shape to cylindrical, as shown in Figure $2 \mathrm{~b}$.

Transition of IB's shape indicates reduction of a distance between ends of EB. Fittings attached to ends of an EB transfer a mechanical power across the muscle and allow for transversal displacement, without changing the length of the muscle ${ }^{7}$.
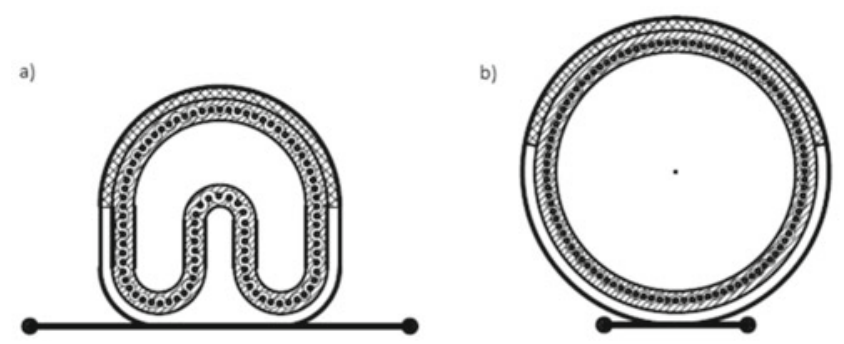

Figure 2. Shape of TPAM a) before inflation of pressured air, b) after inflation

Because of possibility of obtainment a wave motion while using TPAM, they are one of the conception of a drive in a robot used for investigation of human intestines. An additional advantage is the small volume of the muscles. This is significant in case of a rupture of the muscle. While providing pressure of controlled value, the rupture will not cause injury to the patient.

Our goal was obtaining TPAM made of biocompatible materials, which also meet expectations providing proper functioning of the muscles. Recent advances in biomaterials and robotics are providing new opportunities for a variety of biological and medical applications. One of the most useful materials in this field of application is poly(dimethylsiloxane) (PDMS). The popularity of PDMS results from its desirable properties. The highly crosslinked PDMS exhibits thermal stability and great chemical inertness. Consequence of PDMS chemical inertness is biocompatibility and non-toxicity. Equally important are tunable mechanical properties (dependent on the mixing ratio with cross-linking agent), easy fabrication, relative low cost and commercial availability of the PDMS. Furthermore, PDMS has more advantages, such as homogeneous and isotropic properties, porous structure and hydrophobic surface.

PDMS attractiveness also originates from possibilities of easy modification of its surface. These methods can be categorized into three groups: gas-phase methods (including plasma oxidation, ultraviolet irradiation, chemical vapor deposition and metal compounds sputter coating), wet chemical methods (including deposition layer-by-layer, silanization, sol-gel coatings, dynamic modification with surfactants and protein adsorption) and a combination of both ones. They allow to modify PDMS in various ways and obtain more specific materials for different applications ${ }^{\mathbf{8}}$.

PDMS is added to increase the biocompatibility of other polymers. Chaffin et al. used segmented polyurethane multiblock polymers containing PDMS and polyether soft segments to form tough and easily processed thermoplastic elastomers (PDMS-urethanes), which are utilized in medical devices such as leads of defibrillator or pacemaker'.

PDMS is also attractive material for robotics. PDMS with $3 \%$ content of glass bubbles for neutral buoyancy was used to made passive membrane on trailing edges of pectoral fins in manta ray robot ${ }^{10}$. Another bio-inspired robot is gecko-like climbing robot, which has PDMS adhesive footpads ${ }^{\mathbf{1 1}}$.

There are also a few works about pneumatic or hydraulic actuators made of PDMS. Martinez et al. used PDMS with Ecoflex 00-30 (another silicone elastomer) to form composite for PAM, which work as robotic tentacle. PDMS being more rigid than Ecoflex, was more suitable for internal core with pneumatic channel ${ }^{\mathbf{1 2}}$. Another PDMS pneumatic actuator was used for preparation of pneumatic micro fingers, which were parts of micro hand. The fingers were made of two PDMS sheets with balloon chamber between them. Swelling of the chamber generates bending motion of fingers ${ }^{\mathbf{1 3}}$. Another actuator - microgripper using electro-rheological fluid (ERF) was developed by Yoshida et al. It also contains PDMS movable chamber, which was chosen because of PDMS chemical robustness against working fluids and flexibility ${ }^{\mathbf{1 4}}$.

Another polymer, which shows biological and chemical inertness is poly(ethylene terephthalate) (PET). Studies of PET fibers also prove their good thermal stability and mechanical properties. PET fibers melting transition temperature is $245^{\circ} \mathrm{C}$ and its tensile modulus reaches a value of $2.21 \mathrm{GPa}^{15}$. These properties make PET fibers interesting solution for reinforcement in composite materials. Examples of PET reinforcement applications are flexure layer in hexapod crawling robot $^{16}$ and $0.2 \mathrm{~mm}$ frame of wings in flapping robots ${ }^{17}$. PET fibers are also used as reinforcement in pneumatic actuators such PneuFlex ${ }^{18}$. PneuFlex is put inside PET reinforcement helix, which keeps constant diameter of actuator. Interesting results were obtained for fatigue life tests, which showed PET bladder with latex for pneumatic actuators $\left(\mathrm{CleanCut}^{\circledR}\right)$ is able to withstand more than 10000 cycles $^{\mathbf{1 9}}$.

This paper focuses on achieving relevant information about particular properties of the laminate, which is made of PDMS and PET satin (PDMS/PET). Chemical resistance to solutions of various $\mathrm{pH}$ and thermal characteristics (obtained using Vicat apparatus) of three compositions with different amount of cross-linker were examined. Changes in chemical structure of samples (after PET impregnation with PDMS and chemical exposition) 
were investigated by FT-IR spectroscopy. Obtained data allowed to evaluate utility of PDMS/PET composite for TPAM and to choose composition of PDMS with optimal amount of TEOS for the best composite biocompatibility. Also contraction tests for TPAMs (made from PDMS/ PET in three different ways) were conducted.

\section{MATERIAL}

PDMS of analytical grade with viscosity 50.000 cst and tetraethyl orthosilicate (TEOS) of reagent grade were supplied from Aldrich, USA. PET satin was purchased from Jiaxing Dongtai Textile Factory, China. All reagents for the preparation of simulated body fluid (SBF), simulated stomach acid (SSA) and solution of sodium bicarbonate with $\mathrm{pH}$ of pancreatic juice (SPJ) - $\mathrm{NaCl}, \mathrm{NaHCO}_{3}, \mathrm{KCl}, \mathrm{K}_{2} \mathrm{HPO}_{4} \cdot 3 \mathrm{H}_{2} \mathrm{O}, \mathrm{MgCl}_{2} \cdot 6 \mathrm{H}_{2} \mathrm{O}$, $\mathrm{HCl}, \mathrm{CaCl}_{2}, \mathrm{Na}_{2} \mathrm{SO}_{4}$ and 2-amino-2-hydroxymethyl-propane-1.3-diol - were supplied from Aldrich, USA of reagent grade. Ethyl 2-cyanopropenoate for gluing parts of muscle was purchased under trade name Loctite 495 (Henkel, Germany).

\section{EXPERIMENTAL}

Preparation of PDMS/PET samples for biocompatibility tests (chemical resistance, thermal characteristics and FT-IR)

Samples for biocompatibility tests were designed to meet tests expectations and simulate position of layers from our TPAMs. PDMS was mixed with TEOS in different weight ratio $(100: 2.6,100: 5,100: 10)$ and poured into a stainless steel molds (mold size $-10.0 \pm 0.2 \mathrm{x}$ $10.0 \pm 0.2 \times 5.0 \pm 0.2 \mathrm{~mm}$ ) to level of $2.5 \pm 0.2 \mathrm{~mm}$. The appropriate square pieces $(10.0 \pm 0.2 \times 10.0 \pm 0.2$ $\mathrm{mm}$ ) of PET satin (thickness $-0.18 \pm 0.02 \mathrm{~mm}$, weight $0.015 \pm 0.003 \mathrm{~g}$ ) were placed in each mold, when PDMS matrixes become sufficiently viscous to maintain PET satin pieces on matrix's surface (after 30-40 minutes). Rest of PDMS:TEOS mixtures was poured into mold to reach maximum depth of mold. PET satin was also impregnated with PDMS/TEOS mixture before sealing. Cross-linking of PDMS matrix was carried out in standard ambient temperature and pressure. Samples were removed from molds after $24 \mathrm{~h}$. Shape of obtained samples is shown in Figure 3. Optical microscopy image of prepared sample's cross-section is shown in Figure 4.

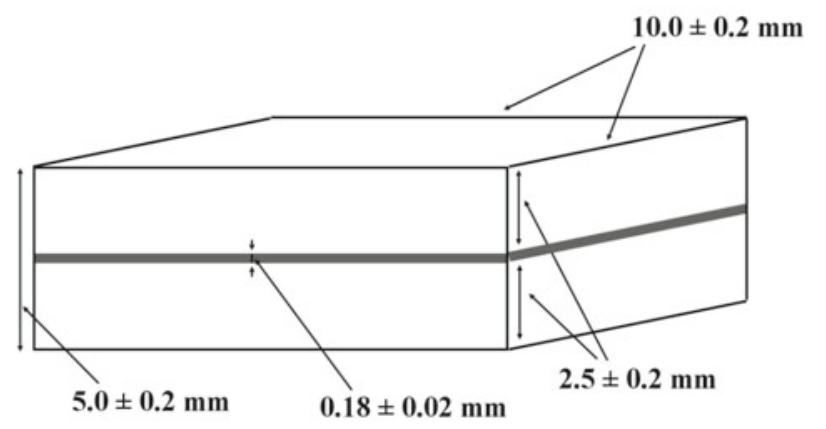

PET satin

PDMS

Figure 3. Shape of sample for biocompatibility tests

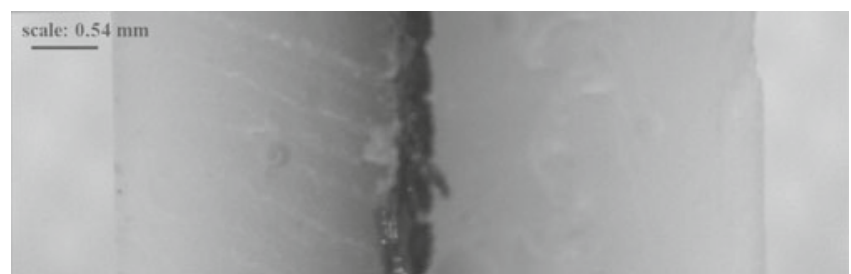

Figure 4. Optical microscopy image of sample's cross-section

\section{Chemical resistance}

The chemical resistance of PDMS/PET was studied using ASTM D 543-87 method ${ }^{21}$. Three solutions of different $\mathrm{pH}$ were prepared to simulate fluids in human body. SSA is a solution of hydrochloric acid, adjusted to $\mathrm{pH} 1.5$, which imitate stomach acid. SPJ adjusted to $\mathrm{pH} 8.4$ allows to estimate influence of basic pancreatic juice. SBF based on Ohtsuki et al. recipe ${ }^{22}$ simulates ion concentration of human blood plasma.

PDMS/PET samples were weighed and immersed in the mentioned above solutions. After 48 hours of exposition to the selected chemicals, samples were dried and weighed again. The percentage of weight change was determined using the following equation:

Weight changes for each combination of PDMS/ TEOS in each solution was measured for six samples and averaged.

\section{Thermal characteristics}

Thermal characteristics were obtained using HDTVICAT 3-300 apparatus (Zwick/Roell, Germany) with CC-300BX bridge thermostat (Huber, Germany). Thermal characteristics were measured according to ISO 306 standard for Vicat softening temperature (VST), using method A120 (with $10 \mathrm{~N}$ load and heating rate $120^{\circ} \mathrm{C} / \mathrm{h}$ ). VST measurement is based on the determination of temperature, which allows blunt, steel needle with circular cross section $1 \mathrm{~mm}^{2}$ to penetrate the test sample to a depth of $1 \mathrm{~mm}$ under defined load. Recommended thickness of the samples should be between 3 and $6.5 \mathrm{~mm}$. Our samples were $5.0 \pm 0.2 \mathrm{~mm}$ thick with PET satin reinforcement at the depth of $2.5 \pm 0.2 \mathrm{~mm}$ from penetrated surface of sample. Position of PET satin was selected to avoid needle stoppage in reinforcement (for depth lower than $1 \mathrm{~mm}$ ). Thermal characteristics in the range $30-130^{\circ} \mathrm{C}$ for six samples for each combination of PDMS/TEOS were averaged. Also reference samples made of cross-linked PDMS matrix without PET reinforcement were prepared according to recipe of preparation samples for biocompatibility tests.

\section{Fourier transform infrared spectroscopy (FT-IR)}

The FT-IR spectra were obtained by using FT-IR 175C spectrophotometer (BIORAD, USA) in an air atmosphere. Spectra of cross-linked PDMS and PET satin taken from composite was measured before and after immersion in the solutions. Also spectrum of PET satin was measured before impregnation with PDMS/ TEOS mixture to investigate effect of the impregnation.

\section{Preparation of muscles}

Obtaining a required shape of muscle is one of the most important elements of muscle production. It is necessary to get a proper U-like shape of inside braid 
and prevent from cleft of material. To achieve a final shape of the muscle, it is needed to create a closed round shaped inside braid at first. It is necessary for accessible placement in a mold, to obtain U-shaped inside braid. Without a pre-preparation, size of muscles will impede a proper production process. Achieving a closed round shape of muscle was possible by creating an overlap form the ends of the inside braid, as shown in Figure 6. The advantage of that solution is strengthening the construction and preventing ruptures. There were two methods of creating the overlap - by imposing PDMS layer between the ends, or by connecting the ends of the inside braid with a ethyl 2-cyanopropenoate glue.

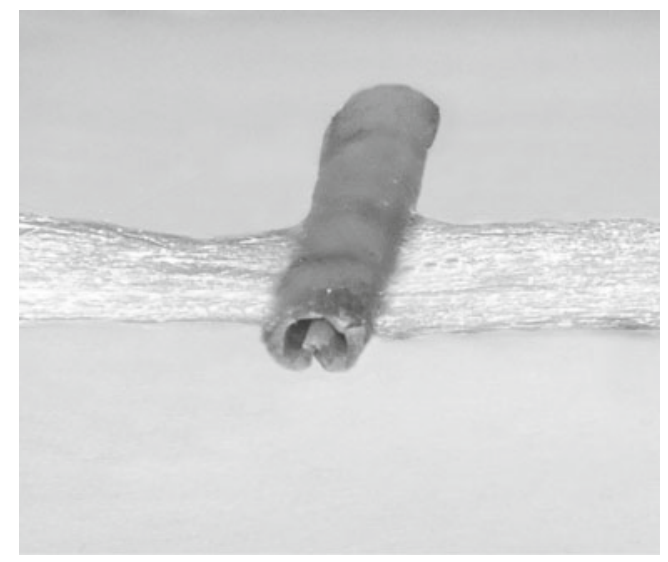

Figure 5. Final shape of the muscle

Moreover, the construction of the muscle has to protect against uncontrolled displacement of inside braid. To obtain a durable connection between inside and outside braid, braids need to be attached to each other. It was obtained in a mold, by imposing another PDMS layer between braids, after a process of covering braids by PDMS and before closing of the mold.

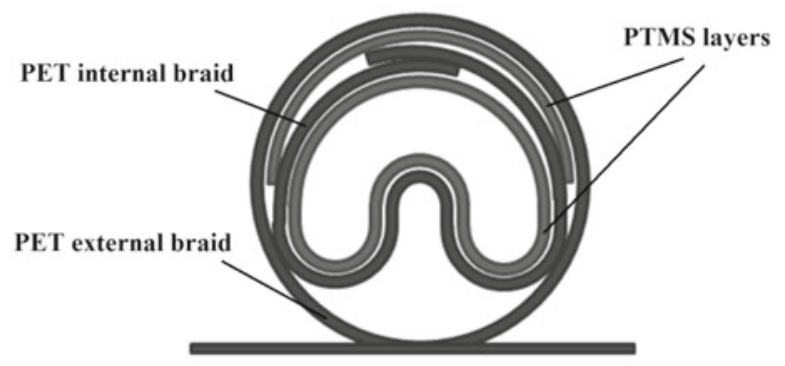

Figure 6. TPAM with an overlap

The second construction was uniform - it did not contain a division into inside and outside braid. The muscle was produced from one piece of material. Overlap of inside braid and connection to outside braid were obtained by plait of the braid material, as shown in Figure 6 . This solution provides tightness of the construction, and precludes uncontrolled displacement of inside braid. Moreover, the implementation of this structure is easier and ensures proper PDMS impregnation of braids material.

\section{Static load characteristics}

There were conducted an experimental research of contraction for presented constructions of muscles to choose more effective structure. There were used muscles

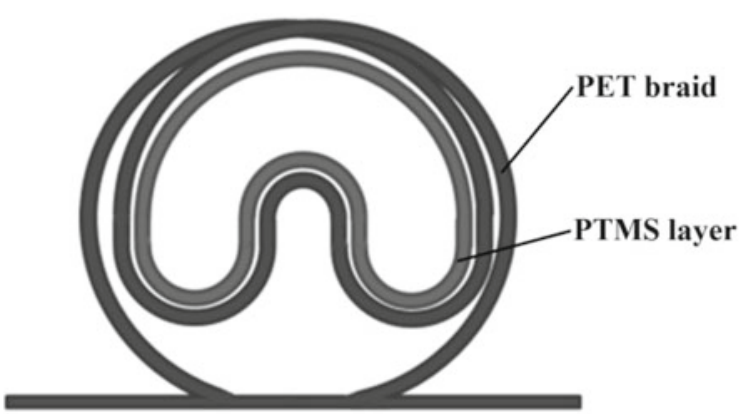

Figure 7. Uniform construction of TPAM

of size $2.5 \mathrm{~mm}$ in diameter and $10 \mathrm{~mm}$ in length. The contraction of a TPAM was determined by the pressure level, the external load and the volume-to-length change of the muscle. Due to that, there was executed a research of references between force $F$ achieved by changing a shape of a muscle and a shrinkage of a muscle h, while the value of pneumatic pressure was constant and equal to $\mathrm{P}=0.1 ; 0.2 ; 0.3 ; 0.4 \mathrm{MPa}$. For each value of a pressure, there were fulfilled the examination of a value of a force which is generated by the muscle by tensioning the EB. For each sample there were fulfilled three tests. Based on a registered data, static load characteristics were plotted.

\section{RESULTS AND DISCUSSION}

\section{Chemical resistance of PDMS/PET}

We compared averaged weight changes for nine different combinations of sample composition and type of solution (Table 1). This comparison was made to ensure, that PDMS/PET is resistant to solutions found in human gastrointestinal tract.

All samples show very good or excellent chemical resistance for SSA, SBF and SPJ. None of weight gain/ loss is greater than $0.7 \%$ of original sample weight. It is very outstanding result due to the fact, that, in real applications, the material will not be exposed to interaction with examined solutions for so long time. Larger average weight changes, observed for samples with PDMS:TEOS (100:10), are probably a consequence of TEOS excess in PDMS matrix, which made that composition more vulnerable for the water solutions. Despite the fact, that chemical resistance of PDMS:TEOS (100:10) is still at an acceptable level, this composition will be rejected in further studies, to achieve the highest possible level of material bioresistance.

\section{Thermal characteristics}

Thermal characteristics of all compositions in the range $30-130^{\circ} \mathrm{C}$ (Fig. 8) was investigated. This range is wider than necessary one, but it was extended beyond the standard temperatures of human body and the highest temperature occurring during work of artificial muscles, to allow the reasonable estimation of the border conditions for the working composite. The obtained results were compared with the results for reference sample without PET satin.

Pure PDMS:TEOS matrix shows acceptable thermal resistance $\left(54^{\circ} \mathrm{C}\right)$ for human body temperature range. The presence of PET satin in composite greatly improves its thermal resistance. This observation is in agreement 
Table 1. Comparison of ranges of weight changes and average weight changes (gain $(+) /$ loss $(-)$ ) for three PDMS/PET samples with different compositions of PDMS cross linked with TEOS and immersed for $48 \mathrm{~h}$ in three different solutions

\begin{tabular}{|c|c|c|c|c|c|}
\hline \multirow{2}{*}{ Simulated fluids $\downarrow$} & \multicolumn{3}{|c|}{ PDMS:TEOS weight ratio } & & \\
\hline & $100: 2.6$ & $100: 5$ & 100:10 & & \\
\hline \multirow{2}{*}{$\begin{array}{l}\text { Simulated stomach acid } \\
\text { (SSA) }\end{array}$} & $-0.148 \%-0.000 \%$ & $-0.170 \%-0.091 \%$ & $-0.524 \%--0.155 \%$ & Range & \multirow{6}{*}{ 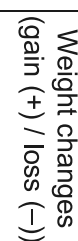 } \\
\hline & $-0.061 \%$ & $0.015 \%$ & $-0.410 \%$ & Average & \\
\hline \multirow{2}{*}{$\begin{array}{l}\text { Simulated body fluid } \\
\text { (SBF) }\end{array}$} & $0.000 \%-0.057 \%$ & $-0.194 \%-0.000 \%$ & $-0.483 \%--0.304 \%$ & Range & \\
\hline & $0.018 \%$ & $-0.090 \%$ & $-0.385 \%$ & Average & \\
\hline \multirow{2}{*}{$\begin{array}{l}\text { Solution of sodium } \\
\text { bicarbonate with } \mathrm{pH} \text { of } \\
\text { pancreatic juice (SPJ) }\end{array}$} & $-0.076 \%-0.063 \%$ & $-0.356 \%-0.078 \%$ & $-0.620 \%--0.181 \%$ & Range & \\
\hline & $-0.025 \%$ & $-0.034 \%$ & $-0.456 \%$ & Average & \\
\hline
\end{tabular}

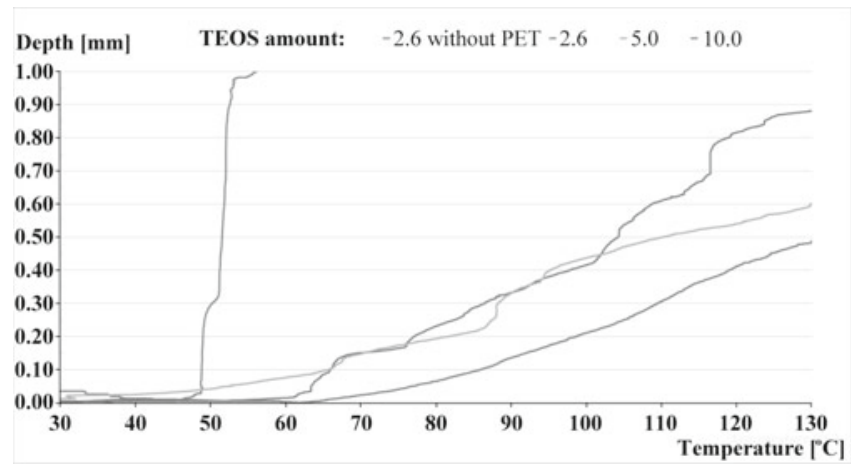

Figure 8. Thermal characteristics of compositions with different TEOS amount compared to reference composition without PET satin

with the material softening temperature, which is about $230240^{\circ} \mathrm{C}^{23}$. PET satin works also as scaffold, which forbid the needle penetration and helps laminate to stay hermetic. Risk of muscle damage in human gastrointestinal tract is very low, but in rare case PDMS:TEOS matrix can be softened and damaged at external side. Presence of PET satin protects internal side, consequently material is still able to work as pneumatic muscle. Higher amount of TEOS in PDMS matrix results in higher thermal resistivity of laminate. Summarizing, all compositions with PET satin show VST higher than $130^{\circ} \mathrm{C}$ and have thermal characteristics appropriate for usage in artificial muscles.

\section{FT-IR}

PDMS:TEOS (100:5) shows the most promising properties, consequently this composition immersed in SBF was chosen as representative sample for FT-IR investigation. FT-IR spectrum of untreated PDMS:TEOS (100:5) was used as reference sample. Figure 9 shows comparison of the spectra.
In both spectra, all characteristic absorption bands for PDMS were observed: $\mathrm{CH}_{3}$ rocking in $\mathrm{Si}-\mathrm{CH}_{3}\left(785 \mathrm{~cm}^{-1}\right)$, Si-O-Si $\left(1008 \mathrm{~cm}^{-1}\right), \mathrm{CH}_{3}$ symmetric bending in $\mathrm{Si}-\mathrm{CH}_{3}$ $\left(1258 \mathrm{~cm}^{-1}\right)$ and $\mathrm{C}-\mathrm{H}$ stretching in $\mathrm{CH}_{3}\left(2962 \mathrm{~cm}^{-1}\right)^{24}$. Similarity of the spectra was measured using QCheck tool (OMNIC software). QCheck degree of similarity between the spectra was 0.9964. It confirm excellent chemical resistance of PDMS:TEOS (100:5) matrix.

Another FTIR spectra, compared to each other, are untreated PET satin and PET satin after PDMS:TEOS (100:5) impregnation (Fig. 10).

The main absorption bands of untreated PET satin are: aliphatic $\mathrm{C}-\mathrm{H}$ antisymmetric stretching vibration $\left(2968 \mathrm{~cm}^{-1}\right), \mathrm{C}=\mathrm{O}$ stretching vibration $\left(1712 \mathrm{~cm}^{-1}\right)$, aromatic skeletal stretching vibration $\left(1409 \mathrm{~cm}^{-1}\right)$, ester group stretching vibration $\left(1258 \mathrm{~cm}^{-1}\right)$, an aromatic substitution pattern and 1.4 substitution bands in the skeletal ring (1097 and $\left.1018 \mathrm{~cm}^{-1}\right)$, ethylene glycol segment $\mathrm{O}-\mathrm{CH}_{2}$ stretching vibration $\left(971 \mathrm{~cm}^{-1}\right)$ and out-of-plane deformation of two carbonyl groups substituted on the aromatic ring $\left(721 \mathrm{~cm}^{-1}\right)^{25}$. Also $1341 \mathrm{~cm}^{-1}$ band (trans conformation of $\mathrm{C}-\mathrm{C}$ bond in $\mathrm{OCH}_{2} \mathrm{CH}_{2} \mathrm{O}$ - moiety) carries information about predominance of crystalline regions in the PET satin ${ }^{\mathbf{2 6}}$.

Differences between spectra of untreated and impregnated PET satin proves presence of PDMS. They are increased intensities of peaks: $2963 \mathrm{~cm}^{-1}$ (C-H stretching in $\left.\mathrm{CH}_{3}\right), 1258 \mathrm{~cm}^{-1}\left(\mathrm{CH}_{3}\right.$ symmetric bending in $\mathrm{Si}-\mathrm{CH}_{3}$, which covers band of $\mathrm{C}(\mathrm{O})-\mathrm{O}$ stretching of ester group), $1093 \mathrm{~cm}^{-1}$ and $1018 \mathrm{~cm}^{-1}$ (Si-O-Si, which cover bands of an aromatic substitution pattern and 1.4-substitution bands in the skeletal ring at 1097 and $1018 \mathrm{~cm}^{-1}$ ). Also new peak, related to PDMS, was observed at 793 $\mathrm{cm}^{-1}\left(\mathrm{CH}_{3}\right.$ rocking in $\left.\mathrm{Si}-\mathrm{CH}_{3}\right)$. Decreased intensities of peaks: $1712 \mathrm{~cm}^{-1}(\mathrm{C}=\mathrm{O}$ stretching vibration $), 1409 \mathrm{~cm}^{-1}$ (aromatic skeletal stretching vibration) and $1341 \mathrm{~cm}^{-1}$ (trans conformation of C-C bond in $\mathrm{OCH}_{2} \mathrm{CH}_{2} \mathrm{O}$ - moiety)

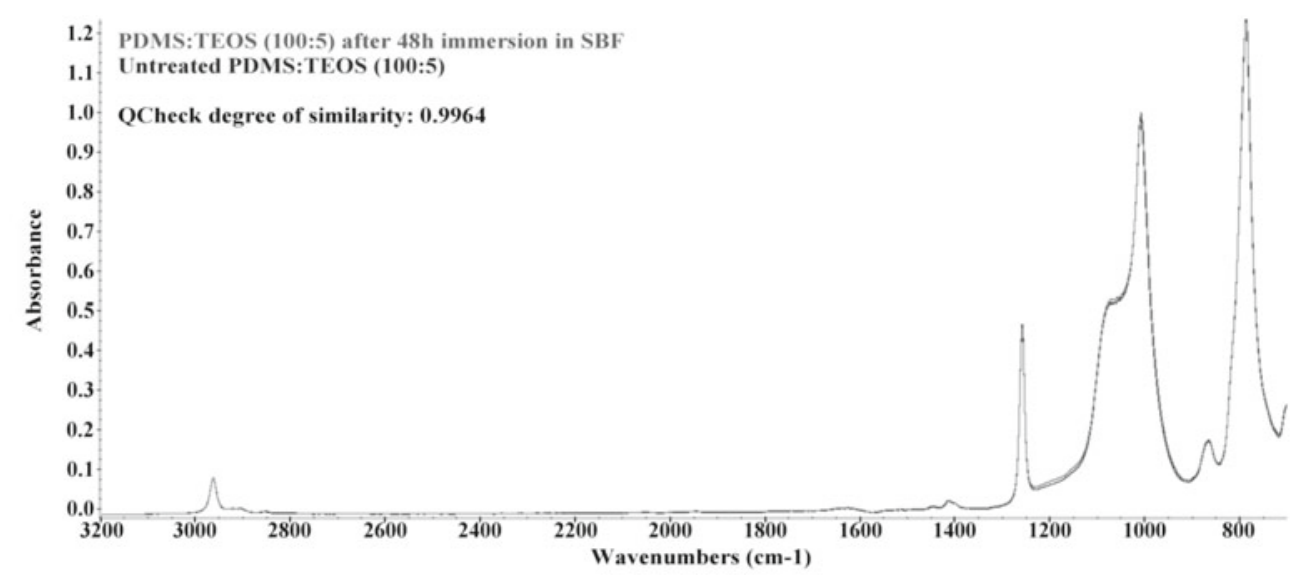

Figure 9. FT-IR spectra comparison of untreated PDMS:TEOS (100:5) with PDMS:TEOS (100:5) after 48 h immersion in SBF 


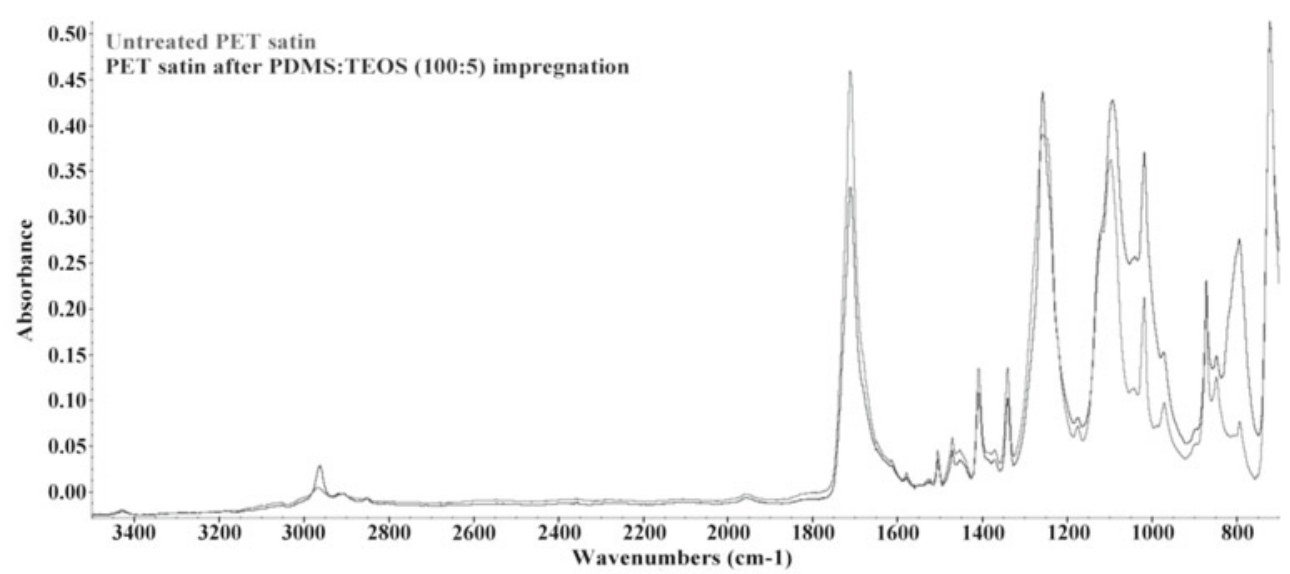

Figure 10. FT-IR spectra comparison of untreated PET satin with PET satin after PDMS:TEOS (100:5) impregnation

reveals interaction of methyl groups from PDMS with carbonyl and aromatic groups from $\mathrm{PET}^{27}$ and probably leads to destruction of -O- $\mathrm{CH}_{2}-\mathrm{CH}_{2}-\mathrm{O}$ - moieties.

Impregnated PET satin was compared to the same sample, which was also immersed in SBF (Fig. 11).

QCheck degree of similarity between the spectra of was 0.9778 . This result still allows to mark PET satin as material with high bioresistance, especially since differences in spectra originates from further PDMS:TEOS impregnation of PET, caused by SBF solution. Similar decreases and increases of peaks are observed, although they occur to a smaller extent.

\section{Static load characteristics}

The Figure 12 presents static characteristic of PAM with overlap of core and PDMS (PDMS core). The muscle deforms easily and small values of pressure are necessary to obtain high values of contraction. For pressure $0.1 \mathrm{MPa}$ the shrinkage is $39 \%$ and for $0.4 \mathrm{MPa}-55 \%$. The force generated by muscle is small - maximum 5.9 $\mathrm{N}$ for $0.4 \mathrm{MPa}$.

Higher values of forces are achieved for TPAM with overlap of core glued with ethyl 2cyanopropenoate (glued core), which static characteristic is shown in Figure 13. The higher rigidity, which enables obtaining higher values of forces, also affects contraction, when the values of shrinkage are $36 \%$ for $0.1 \mathrm{MPa}$ and $41 \%$ for $0.4 \mathrm{MPa}$.

In Figure 14, there is presented a static characteristic for uniform TPAM. Noticeable is, that this construction allows obtaining higher values of pressure than TPAM with glued core (from $39 \%$ for $0.1 \mathrm{MPa}$, to $46 \%$ for $0.4 \mathrm{MPa}$ ), but generated forces similar to this solution and much higher than TPAM with PDMS core.

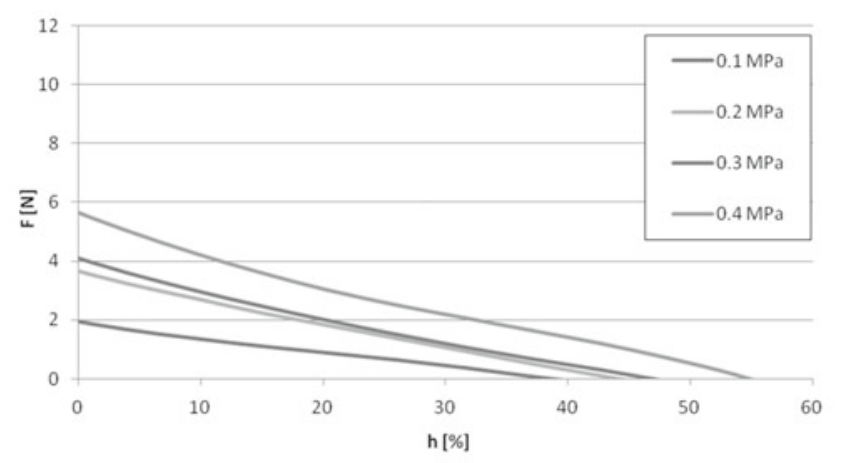

Figure 12. Static characteristic of TPAM with PDMS core

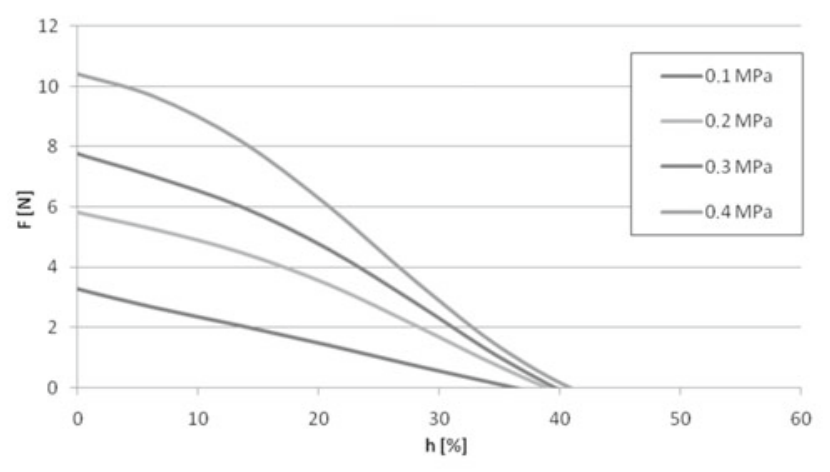

Figure 13. Static characteristic of TPAM with glued core

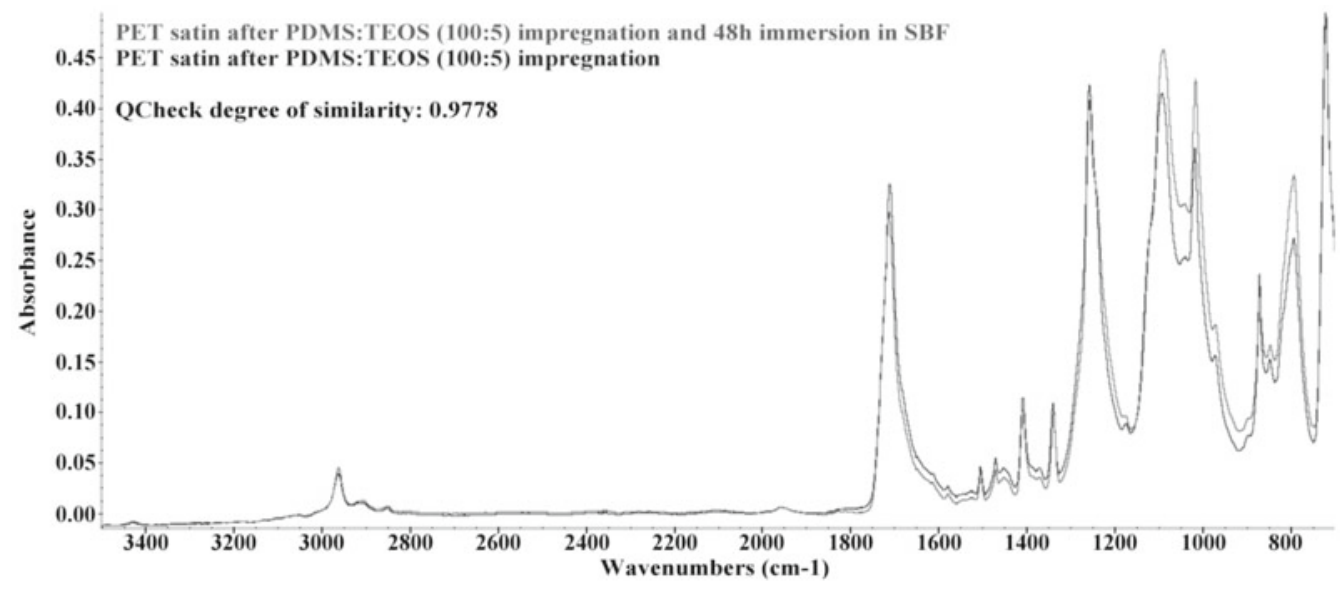

Figure 11. FT-IR spectra comparison of PET satin after PDMS:TEOS (100:5) impregnation with PET satin after PDMS:TEOS (100:5) impregnation and $48 \mathrm{~h}$ immersion in SBF 


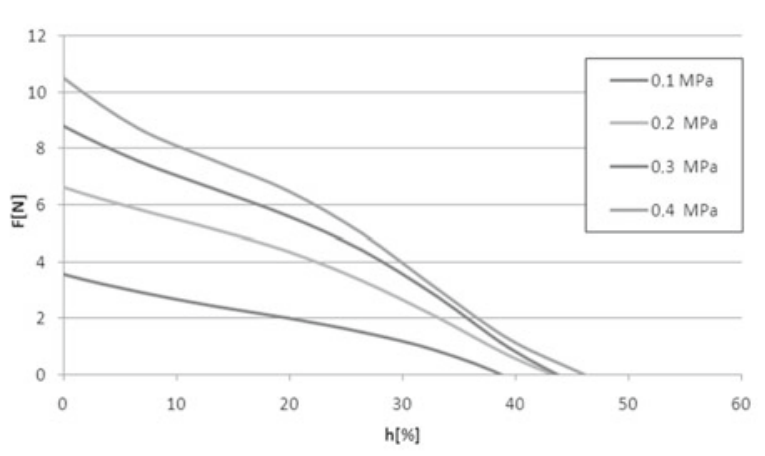

Figure 14. Static characteristic of uniform TPAM

\section{CONCLUSIONS}

PDMS/PET performs perfectly as biocompatible composite. Biocompatibility was proved in bioresistance aspect (chemical resistance, FT-IR) and thermal-mechanical aspect (thermal and static load characteristics). While PDMS is responsible for chemical resistance and tightness, PET works as mechanical and thermal reinforcement. The best composite in the field of biocompatibility is PDMS/PET with PDMS:TEOS weight ratio 100:5. Its FT-IR spectra allowed to investigate mechanism of impregnation and chemical resistance more closely. Results of the investigation did not undermine biocompatibility of the composite.

The construction of the muscle affects values of force and shrinkage obtained while filling muscle with compressed air. In solutions with overlap, the technology impacts on results, so it is possible to achieve high values of forces or shrinkage. The best results are achieved with last solution, Uniform TPAM, where high values of forces and shrinkage are achieved at the same time. Moreover, this solution provides tightness of the construction, what precludes uncontrolled displacement of inside braid or rupture of muscle.

TPAMs, because of their structure, size and biocompatibility, can be considered for use in robots for medical applications.

\section{LITERATURE CITED}

1. Daerden, F. \& Lefeber, D. (2002). Pneumatic artificial muscles: actuators for robotics and automation. Eur. J. Mech. Environ. Eng. 47(1), 10-21.

2. Chou, C.P. \& Hannaford, B. Measurement and modeling of McKibben Pneumatic Artificial Muscles. (1996). IEEE Trans Robot Autom. 12(1), 90-102. DOI: 10.1109/70.481753.

3. Daerden, F. \& Lefeber, D. (2001). The concept and design of Pleated Pneumatic Artificial Muscles. Int. J. Fluid. Power. 2(3), 41-50. DOI: 10.1080/14399776.2001.10781119.

4. Villegas, D., Van Damme, M., Vanderborght, B., Beyl, P. \& Lefeber, D. (2012). Third-Generation Pleated Pneumatic Artificial Muscles for Robotic Applications: Development and Comparison with McKibben Muscle. Adv. Robot. 26(11-12), 1205-1227. DOI: 10.1080/01691864.2012.689722.

5. Lee, Y.K. \& Shimoyama, I. (2002, January). A multi-channel micro valve for micro pneumatic artificial muscle. In Micro Electro Mechanical Systems, 2002. The Fifteenth IEEE International Conference on (pp. 702-705). IEEE.

6. Lee, Y.K. \& Shimoyama, I. (1999). A skeletal framework artificial hand actuated by pneumatic artificial muscles. In Robotics and Automation, 1999. Proceedings. 1999 IEEE International Conference on (Vol. 2, pp. 926-931). IEEE.
7. Koter, K., Podsedkowski, L. \& Szmechtyk, T. (2015, July). Transversal Pneumatic Artificial Muscles. In Robot Motion and Control (RoMoCo), 2015 10th International Workshop on (pp. 235-239). IEEE. DOI: 10.1109/RoMoCo.2015.7219741. 8. Zhou, J., Ellis, A.V. \& Voelcker, N.H. (2010). Recent developments in PDMS surface modification for microfluidic devices. Electrophoresis 31(1), 2-16. DOI: 10.1002/elps.200900475.

9. Chaffin, K.A., Wilson, C.L., Himes, A.K., Dawson, J.W., Haddad, T.D., Buckalew, A.J. \& Simha, N.K. (2013). Abrasion and fatigue resistance of PDMS containing multiblock polyurethanes after accelerated water exposure at elevated temperature. Biomaterials 34(33), 8030-8041. DOI: 10.1016/j. biomaterials.2013.06.049.

10. Chen, Z., Um, T.I. \& Bart-Smith, H. (2012). Bio-inspired robotic manta ray powered by ionic polymer-metal composite artificial muscles. Int. J. Smart. Nano. Mat. 3(4), 296-308. DOI: 10.1080/19475411.2012.686458.

11. Unver, O., Uneri, A., Aydemir, A. \& Sitti, M. (2006, May). Geckobot: a gecko inspired climbing robot using elastomer adhesives. In Robotics and Automation, 2006. ICRA 2006. Proceedings 2006 IEEE International Conference on (pp. 2329-2335). IEEE.

12. Martinez, R.V., Branch, J.L., Fish, C.R., Jin, L., Shepherd, R.F., Nunes, R. \& Whitesides, G.M. (2013). Robotic tentacles with three-dimensional mobility based on flexible elastomers. Adv. Mater. 25(2), 205-212. DOI: 10.1002/adma.201203002. 13. Konishi, S., Nokata, M., Jeong, O.C., Kusuda, S., Sakakibara, T., Kuwayama, M. \& Tsutsumi, H. (2006, May). Pneumatic micro hand and miniaturized parallel link robot for micro manipulation robot system. In Robotics and Automation, 2006. ICRA 2006. Proceedings 2006 IEEE International Conference on (pp. 1036-1041). IEEE. DOI: 10.1109/ROBOT.2006.1641846. 14. Yoshida, K., Ide, T., Kim, J.W. \& Yokota, S. (2009, August). A microgripper using electro-rheological fluid. In ICCAS-SICE, 2009 (pp. 2987-2990). IEEE.

15. Chang, J.H., Kim, S.J., Joo, Y.L. \& Im, S. (2004). Poly (ethylene terephthalate) nanocomposites by in situ interlayer polymerization: the thermo-mechanical properties and morphology of the hybryd fibers. Polymer, 45(3), 919-926. DOI: 10.1016/j.polymer.2003.11.037.

16. Hoover, A.M. \& Fearing, R.S. (2008, May). Fast scale prototyping for folded millirobots. In Robotics and Automation, 2008. ICRA 2008. IEEE International Conference on (pp. 886-892). IEEE.

17. Ming, A., Luekiatphaisan, N. \& Shimojo, M. (2012, August). Development of flapping robots using piezoelectric fiber composites-Improvement of flapping mechanism inspired from insects with indirect flight muscle. In Mechatronics and Automation (ICMA), 2012 International Conference on (pp. 1880-1885). IEEE.

18. Deimel, R. \& Brock, O. (2013, May). A compliant hand based on a novel pneumatic actuator. In Robotics and Automation (ICRA), 2013 IEEE International Conference on (pp. 2047-2053). IEEE. DOI: 10.1109/ICRA.2013.6630851.

19. Kingsley, D. \& Quinn, R.D. (2002). Fatigue life and frequency response of braided pneumatic actuators. In Robotics and Automation, 2002. Proceedings. ICRA'02. IEEE International Conference on (Vol. 3, pp. 2830-2835). IEEE. DOI: 10.1109/ROBOT.2002.1013661.

20. Loganathan, K.S. (1998). Rubber engineering. Indian Rubber Institute, McGraw-Hill, New York, Chapter 1(2000).

21. American Society for Testing and Materials. (1992). ASTM D 543-87. Standard methods for evaluating the resistance of plastics to chemical reagents. Annual book of ASTM standards 8(1). Philadelphia (PA).

22. Ohtsuki, C., Kokubo, T. \& Yamamuro, T. (1992). Mechanism of apatite formation on $\mathrm{CaO} \mathrm{SiO} 2 \mathrm{P} 2 \mathrm{O} 5$ glasses in a simulated body fluid. J. Non-Cryst Sol. 143, 84-92. DOI: 10.1016/S0022-3093(05)80556-3. 
23. Roff, W.J. \& Scott, J.R. (2013). Fibres, films, plastics and rubbers: a handbook of common polymers. Elsevier.

24. Lee, J., Kim, J., Kim, H., Bae, Y.M., Lee, K.H. \& Cho, H.J. (2013). Effect of thermal treatment on the chemical resistance of polydimethylsiloxane for microfluidic devices. J. Micromech. Microengin. 23(3), 035007. DOI: 10.1088/0960-1317/23/3/035007. 25. Holland, B.J. \& Hay, J.N. (2002). The thermal degradation of PET and analogous polyesters measured by thermal analysis-Fourier transform infrared spectroscopy. Polymer 43(6), 1835-1847. DOI: 10.1016/S0032-3861(01)00775-3.

26. Zhang, Y., Zhang, J., Lu, Y., Duan, Y., Yan, S. \& Shen, D. (2004). Glass transition temperature determination of poly (ethylene terephthalate) thin films using reflection-absorption FTIR. Macromolecules 37(7), 2532-2537. DOI: 10.1021/ ma035709f.

27. Zhang, C. \& Chen, Z. (2013). Probing Molecular Structures of Poly (dimethylsiloxane) at Buried Interfaces in Situ. J. Phys. Chem. C, 117(8), 3903-3914. DOI: 10.1021/jp307472j. 